\title{
Marguerite Andersen et le moi en l'absence de l'autre : De mémoire de femme
}

\author{
Julie Tennier, Université de Toronto
}

Depuis la fin du $\mathrm{XX}^{\mathrm{e}}$ siècle, les recherches menées sur l'autobiographie et l'autofiction soulignent les effets du discours postmoderne sur la notion d'identité, en particulier sur la façon dont on comprend la complexité de l'être qui se forme dans un processus constant de devenir. L'analyse des modalités de la mise en discours du moi nous permet de réfléchir à la construction identitaire et à ses mécanismes de production. Chez Marguerite Andersen, romancière franco-ontarienne d'origine allemande, qui se représente dans quatre livres - le roman «semi-autobiographique »De mémoire de femme, le récit autobiographique Parallèles, la prose poétique Bleu sur blanc et le roman autobiographique Le figuier sur le toit - de même que dans certaines nouvelles des Crus de l'Esplanade ${ }^{1}$, cette construction s'avère être un processus dynamique et créatif de mise au monde de soi. Le rôle actif que se donne l'auteure, les opérateurs d'identification dans le texte et la nature fictive du récit rendent le terme « autofiction » particulièrement approprié dans le contexte d'une analyse de l'œuvre ${ }^{2}$, l'ancrant dans les mouvements et les théories littéraires féministes des années 1970 et 1980 qui, selon Joanne Saul, ont servi à déplacer la réflexion critique des préoccupations thématiques aux considérations formelles de l'autobiographie, plus précisément « to an examination of and an attention to language as a site of power and struggle, to a focus on the body, and to a recognition of woman as the subject rather than the object of the text » (8). L'appropriation du corps de l'écrivaine, dans toute sa complexité subjective, qui s'exprime dans l'acte d'écriture, est placée au premier plan et les textes produits vivent une transformation esthétique. Dans cette étude, nous nous pencherons sur une œuvre en particulier, soit De mémoire de femme, pour explorer comment l'éloignement de différentes figures d'altérité à un moment particulier dans la vie de la protagoniste incite chez elle le désir de se raconter et d'ajuster en quelque sorte son tir en ce qui concerne son identité. La protagonistenarratrice, son discours et son parcours identitaire sont affectés par l'absence de l'«autre » privilégié, c'est-à-dire «celui ou celle avec qui [elle] s'était cru en train de 'partager son existence'», son départ provoquant «une catastrophe structurelle » 
(Landowski 131). À l'aide des théories énoncées par Éric Landowski, Charles Taylor et Julia Kristeva, nous verrons comment l'exercice narratif est mis en branle par le déséquilibre provoqué par l'absence de l'autre que l'écrivaine cherche à combler dans le but de s'épanouir.

Publié en 1982 aux Éditions Quinze, De mémoire de femme remporte l'année suivante le Prix du journal de Montréal. Une deuxième édition revue et corrigée, avec une préface de Lucie Hotte, paraît en 2002 aux Éditions L'Interligne. Ce premier roman de Marguerite Andersen est narré par le personnage principal nommé Anne Grimm. Selon Lucie Hotte, « le jeu entre Grimm et Andersen tient plus de l'ordre du pseudonyme que de l'invention » (12), puisque les deux noms renvoient à de célèbres conteurs. C'est l'un des moyens par lesquels l'auteure subvertit les normes de l'autobiographie et entreprend la représentation de soi de façon ludique. Le projet d'écriture poursuivi par Anne passe par une affirmation de la vie, par «une recherche d'[elle]-même, en quête d'un sens à assigner à [son] propre être au monde » (Landowski 151). Pour ce faire, elle se sert d'un casier d'imprimerie comme «lieu écrit de son passé » (Andersen 25), qui finira par représenter son «[a]utoportrait, Übersicht, mosaïque, vie taillée, cadrée, autrement dit matée »(26). Le casier d'imprimerie prête une forme aux souvenirs de la narratrice (quelque arbitraire et statique qu'elle soit) et la mémoire anime le récit de façon créative. Par « un système simple et hygiénique pour mettre de l'ordre dans [sa] vie » (26), les objets collectionnés comme souvenirs au fil des années sont remplacés par des fiches portant le ou les mots qui les représentent le mieux. Les mots prennent la place des objets, témoignages du désordre et du discontinu, et permettent au récit de prendre forme. Cette mesure offre des effets positifs sur le plan psychologique. Anne se sent «sécurisée dans le fini, dans ce qui a un cadre, un rangement possible » (27). Elle remarque que la structure ordonnée que le cadre impose à son récit est en opposition au mélange d'éléments qui la définissent intérieurement :

Il y aura de l'ordre, un système clair et raisonné, heureux. Mais en moi, hélas ! [...] j'ai trop tricoté et les motifs de mon ouvrage sont tels que les fils, du côté envers, se croisent, s'enroulent l'un autour de l'autre, multicolores, inextricables. Arriverai-je à en retrouver le plan ? Il me faut y regarder de près, me dire, me taire, laisser parler les autres, me chercher, me trouver. (35) 
C'est un exercice d'organisation qui la satisfera grandement grâce aux multiples choix qu'elle fera pour créer le récit de vie qui exprimera son «identité authentique, autodéterminée et auto-règlementée » (Hoover Braendlin 18). Chaque section du roman permet à la narratrice de « [se] dire, [se] taire, laisser parler les autres, [se] chercher, [se] trouver » (Andersen 35) et, dans son ensemble, le texte trace les souvenirs qui forment les motifs de son parcours identitaire, les fondements de son être et l'avenir qu'elle s'imagine.

\section{Le devenir de l'être}

Dans Présences de l'autre, Landowski se penche sur la dynamique qui existe entre le sujet et le groupe de référence dans lequel il évolue, affirmant que «la problématique de l'identité ne relève pas seulement d'une logique de la différence et du discontinu, elle appelle surtout la mise en œuvre d'une sémiotique du continu, du 'devenir' ou, [...] de l'instabilité » (1997 44). Nous pouvons considérer un groupe de référence comme étant une collectivité en fonction de laquelle le sujet se détermine grâce à diverses pressions sociales d'ordre inclusives ou exclusives. Pour le sujet qui se distancie de cette communauté, celle-ci peut représenter une «figure [d'altérité] antithétique »(40). Même chez le sujet qui perçoit le groupe de référence comme un « Nous » dont il fait partie, chaque membre de cette communauté peut remplir la fonction d'objet, devenant pour lui l'autre avec lequel il «établi[t] un juste rapport » (43) qui contribue à son devenir. Les sujets se reconnaissent dans la position qu'ils adoptent en relation au groupe de référence ou à l'autre, ainsi que dans la position dans laquelle ils sont placés par ce groupe (59).

Cette dynamique de groupe fait en sorte que la présence de l'autre, «toute proche ou plus lointaine, chère ou même détestée, peu importe à la limite, [est] une part déterminante de notre vie ». (130) La situation des sujets par rapport à un groupe de référence se fait en fonction de leurs désirs et de leurs intentions de se rapprocher ou de s'éloigner du profil du sujet type idéal qui exemplifie cette communauté (54). Ce sujet idéal est entouré de sujets qui «s'orient[ent] par référence à lui en se signalant du même coup - à raison même de leur acentralité - comme autant d'incarnations originales et distinctes de 'l'Autre' » (53). Chaque individu entre en relation intersubjective avec les 
membres du groupe de référence, agissant en tant que sujet et/ou objet l'un pour l'autre de façon interchangeable. Les sujets adoptent des «'styles de vie' [qui] sont [...], en premier lieu, des projets de vie, mis en acte, et pour cela d'abord choisis sur la base d'une intentionnalité, articulée ou diffuse, qui les fonde, et qu'en retour ils manifestent, apprenant ainsi aux sujets, à travers leur faire et leur devenir, ce qu'ils 'sont' » (58).

Ainsi, si Anne, dans De mémoire de femme, choisit de consacrer un an de sa vie à un projet d'écriture introspectif, c'est dans le but de mieux cibler et comprendre son être du passé, de se situer dans le présent et de choisir son orientation future. Le récit d'Anne montre que les relations interpersonnelles dépassent l'ordre intersubjectif et deviennent « intersomatiques » (2004 78), tel que le suggère Landowski. Vivre la présence sensible de l'autre, et celle de son propre corps, comme objet, permet au sujet de mieux concevoir son être et de continuer à concilier ses idéaux avec son parcours vital ${ }^{3}$. Le corps s'exprime dans un contexte immédiat partagé et le sens est vécu à travers la co-présence et l'interaction avec les sujets qui l'entourent. Qu'arrive-t-il lorsque le ou les sujets coprésents, ce ou ces autres qui nous sont chers, disparaissent? Cette rupture est vécue presque comme une mort, obligeant le sujet à s'identifier en l'absence de l'autre. Selon Landowski, «[...] ce qui vient soudain à nous manquer, c'est l'être [...] à travers lequel s'édifiait notre relation à tous les autres et, en définitive, au monde en général. Ainsi privés [...] de l'intersubjectivité en tant que mode de configuration du monde, nous nous retrouvons alors seul à seul directement face à notre propre être au monde » $(1997,131)$. En l'absence de l'autre, le sujet se trouve dérouté et il doit alors ajuster son devenir en conscience de cause, se mettant en présence de l'être rétrograde de ses souvenirs, cet autre du passé, dans le but de (re)faire sens de son moi et de son être au monde.

De mémoire de femme documente les réflexions de la narratrice sur sa vie, passée et présente, lui permettant de se dire en fonction du groupe de référence qui participe à la construction de son identité et de juger de sa position en relation à celui-ci. Anne constate que les hommes qu'elle a connus et les valeurs patriarcales qu'ils personnifient, l'ont minée et empêchée d'exprimer son moi authentique. Se distanciant de toute figure normative oppressive, le trajet d'Anne s'oriente vers un nouvel idéal identitaire, non pas calqué sur un modèle féminin traditionnel, mais sur une forme inédite qui reflète les désirs, les besoins et les valeurs de la narratrice en tant que femme contemporaine. Le 
texte qu'elle produit illustre comment «[1]a connaissance de soi comporte [...] une profondeur temporelle, [et] inclut le récit », tel que l'affirme Taylor dans Les sources $d u$ moi : La formation de l'identité moderne (75). Selon lui, c'est à travers une narration cohérente que l'être arrive à comprendre qui il est, comment il l'est devenu et ce qu'il souhaite devenir (71-72). Cette compréhension de soi est en transformation constante, le passage du temps rendant désuète chaque ancienne version du soi conçue chez l'être qui souhaite que «l'avenir 'rachète' le passé, qu'il fasse partie du récit d'une vie qui ait un sens et une raison d'être » (76). L'être atteint un sens de plénitude dans l'accomplissement des gestes quotidiens qu'il perçoit comme étant dignes de sa personne, ce qui ajoute un élément de discrimination qualitative au processus de sémantisation identitaire et ainsi, pour avoir une identité, l'être a « besoin d'une orientation vers le bien, donc d'un certain sens des discriminations qualitatives, de ce qui est incomparablement supérieur » (71). Enfin, Taylor précise que «[1]a pleine définition de l'identité d'un individu inclut $[. .$.$] habituellement non seulement sa position relativement aux questions$ morales et spirituelles, mais aussi une référence à une communauté qui la détermine » (57).

Voyons comment la narratrice se situe au sein de sa communauté référentielle. Anne Grimm présente son projet d'écriture dans la première partie du roman, la seule à ne pas porter de titre. Écrite sous forme diaristique, cette introduction nous situe sur le plan spatio-temporel à Paris au moment de la rédaction du roman et nous présente les inquiétudes de l'auteure quant à la méthode et à la structure du projet. Anne esquisse ainsi les grandes lignes de son identité :

Née en 1924, dans la partie de l'Allemagne qui est actuellement la République démocratique allemande. Famille bourgeoise. Un grand-père théologien, recteur d'université ; un autre également luthérien, mais missionnaire. Un père fonctionnaire et écrivain, député au Landtag ${ }^{4}$. Ma mère. La mer Baltique. Douce, belle. Deux sœurs plus âgées. Trois maris. Trois enfants, trois langues, une vie vécue sur trois continents. (28)

Les quatre sections suivantes correspondent aux quatre parties du casier d'imprimerie qu'Anne utilise pour encadrer son projet mémoriel et qui comprennent les mots qu'elle y a classés. La première section intitulée «Pré-texte en vingt-deux éléments » (37) mêle les souvenirs d'Anne à la fiction par une adaptation des écrits de son père. Ici, le récit de sa naissance et l'affirmation de son amour pour ses parents mènent à un survol des lieux 
habités pendant son enfance, les relations entretenues avec ces lieux et entre les membres de sa famille. Nous lisons par la suite «Trois paratextes autour de trente-trois personnages » (91) qui regroupe trois narrations présentant les réflexions de Maria, la mère d'Anne, ainsi que celles de sa sœur Emma et de son fils Dominique. Par les observations de ces trois personnages, surtout en ce qui concerne les amours d'Anne, ses déplacements ainsi que son rôle en tant qu'épouse et mère, nous constatons la position qu'elle occupe dans le groupe de référence familial qui marque son identité. Anne « laiss[e] parler les autres » (35) au début du roman pour dévoiler l'effet que leur regard a eu sur son cheminement.

Trois membres de la famille d'Anne, soit sa mère Maria, sa sœur Emma et son fils Dominique, s'expriment au sujet de son comportement. Comme ces personnages figurent dans le «roman » d'Anne, on peut dire qu'elle se projette dans l'esprit de chacun d'eux. Selon Nancy Huston, «se produit alors: cristallisation, essentialisation - une 'précipitation', en quelque sorte, d'affects demeurés jusque-là flous et insaisissables » (159). Dans les trois cas, il s'agit donc, malgré les apparences, d'Anne qui réinterprète les événements. Le premier «paratexte » est une narration omnisciente axée sur le point de vue de Maria, qui décrit le premier et bref mariage d'Anne en y jetant un éclairage nouveau. Cette mère, soucieuse des autres, reconnait les signes d'une femme qui se range dans le mariage et se transforme en fonction de l'idéal patriarcal normatif. Elle ne veut pas voir sa fille cadette, intelligente et insouciante, devenir une femme soumise, comme le sont devenues les deux sœurs aînées d'Anne, mais peut-elle imaginer un avenir différent pour sa fille ? Elle n'a pourtant pas à se soucier longtemps de ce mariage car, trois mois plus tard, Anne lui annonce son désir de quitter Paul.

Le «paratexte » suivant relate les impressions d'Emma, sœur d'Anne, au sujet du comportement de la narratrice suite à son divorce de Paul et pendant son deuxième mariage avec Pierre. Chez Emma, nous retrouvons une attitude plus critique que celle de la mère. Elle a du mal à comprendre sa sœur cadette. Bien qu'elle l'aime et lui souhaite le bonheur, elle la trouve «égoïste » (145) et lui reproche son enfance privilégiée. Emma trouve qu'Anne «manquait de logique, malgré son baccalauréat. Anne énergique, Anne douée, Emma en avait marre. [...] Folle, voilà ce qu'elle était. [...] Ce qu'elle désirait, c'était l'étranger, hors de la famille. Emma lui en voulait. L'Allemagne, la famille ne se 
rejetaient pas ainsi » (122-3). Anne assouvit son désir de l'étranger en épousant Pierre, un Français qu'elle avait rencontré en Allemagne, et qu'elle suit à sa ville natale de Tunis. Mais cela se passe mal. Lorsqu'Emma apprend que sa sœur, maintenant mère de deux enfants, veut quitter son mari qui la bat, elle se demande : «Anne n'exagérait-elle pas ? N'avait-elle pas toujours été exaltée, hystérique ?» (135). Elle trouve Anne ingrate et désinvolte car, pour Emma, le bonheur se retrouve dans l'ordre patriarcal qui exige qu'une femme prenne ce qui lui est donné, s'en contente et se taise. Emma juge sévèrement cette sœur choyée et rebelle, qui semble chercher par tous les moyens une sorte de liberté, surtout dans l'expression de son corps, qui ne se réconcilie pas avec l'idée qu'elle se fait du bonheur 'normal'. Enfin, le regard critique qu'Emma pose sur sa sœur suggère un manque de solidarité entre les deux femmes et marque un écart entre le discours hégémonique familial et le comportement d'Anne.

Le dernier «paratexte » expose les observations de Dominique, fils aîné d'Anne, concernant la relation tumultueuse entre sa mère et son troisième époux, Amédée. $\mathrm{Ce}$ récit, encore moins flatteur que le précédent, présente Anne comme étant «bête » (165), «[a]platie devant un homme » (165) qui a sept ans de moins qu'elle (166). Dominique aime sa mère pour sa force de caractère (207), ce qui rend difficile à comprendre sa subordination volontaire à un homme qui l'exploite et lui manque de respect ${ }^{5}$. Dominique exprime aussi un sentiment d'abandon en décrivant sa relation avec sa mère : « Il faut dire que jusqu'à l'entrée en scène d'Amédée, Maman s'est conduite comme si elle nous aimait, ce n'est qu'après qu'elle a changé » (191). Les narrations de Maria, d'Emma et de Dominique reflètent le regard critique dont Anne a été la cible et, de manière plus significative, les regrets qu'elle ressent en reconnaissant ne pas avoir su répondre aux attentes morales des membres de sa famille qui la jugent «autre». Dans la section suivante, «Avant A. ou vingt-huit questions » (228), Anne ajoute sa voix aux narrations précédentes. Son récit lui permet d'exprimer ses passions, sa sexualité et son corps, ce qui complète les discours antérieurs et dévoile ce que l'acte d'écriture représente pour elle. Ensuite, la partie intitulée «A. » (290) raconte sa relation avec Amédée, l'époux qui l'a le plus marquée et avec qui la rupture est la plus récente et la plus difficile à vivre. En se remémorant cette relation tumultueuse, Anne explore la force de ses passions et les conséquences qu'elles ont eues sur sa vie et ses relations. 


\section{En l'absence de l'autre}

Anne est à la recherche d'une réconciliation identitaire qui lui permettra d'atteindre un état de paix et d'autonomie et, pour ce faire, elle doit se fier à la mémoire familiale, qui est ce sur quoi repose la conception de l'être (Muxel 30). La narratrice avoue : «Le plus difficile, pour moi, c'est de ne pas faire partie d'une cellule familiale traditionnelle. Vater, Mutter, Kind. Un paradis qui, d'après mes expériences, ne me convient aucunement, mais auquel j'ai aspiré pendant longtemps et que je regrette toujours »(341). En tant qu'adulte, Anne tente par trois fois de recréer l'«intimité parfaite » de la famille traditionnelle associée à sa naissance, « [son] début, [son] origine, [son] éden » (42). Dans chaque mariage, Anne est en quête d'une vie heureuse, comblée, et elle croit voir en chaque partenaire une composante nécessaire à la création d'une famille idéale. De ses deux premiers mariages, Anne ne retient aucun souvenir d'intimité. Son premier époux est impuissant ; l'acte sexuel avec l'homme taciturne qui est son deuxième époux n'est qu'un exercice de vigueur masculine toléré patiemment par la narratrice. Amédée se distingue des époux précédents, car il éveille le corps physique et érotique d'Anne si longtemps refoulé. Elle croit s'affranchir dans cette relation, mais ne reconnaît que plus tard son prix excessif. Pour plaire à son amant, Anne se distancie de ses deux fils nés de son deuxième mariage et avoue «qu'au début c'était comme une délivrance » (299) de ne pas se soucier de ses enfants, ce qui deviendra source de honte chez elle. Elle veut un troisième enfant pour créer, avec Amédée, une cellule familiale traditionnelle. Toutefois, Amédée rejette cet idéal : «[Il] m’explique que je ne suis pas de sa famille puisque je ne suis pas de son sang. L'alliance, la vie en commun, l'enfant commun ne comptent pas à ses yeux. 'Tu n'es pas de ma famille.' Exclue. 'Je ne suis pas de la tienne, je n'ai rien à faire avec tes fils.' Marthe, [l'enfant du désir] de sa mère, n'est pour Amédée qu'un trait d'union facultatif » (306). En l'absence de ses parents défunts et par son exclusion du cercle familial d'Amédée, Anne se voit seule et lorsque cet époux la quitte, le lien familial qui assure (tant bien que mal) la cohérence du parcours de la narratrice est rompu. Anne se retrouve dans un état de déséquilibre débilitant. En attendant Amédée sur le seuil du domicile de sa maîtresse, «[Anne] pleure sur ellemême, sur la vie qui est si difficile à vivre, elle aimerait ne plus exister, ne faire qu'un avec le paillasson, se confondre avec le sol, le mur, le bois de la porte. [...] À quoi sert-il 
de vivre, de faire des enfants qui seront malheureux à leur tour, qui eux aussi, un jour, voudront ne pas avoir eu à venir au monde ? »(319). À la suite de cette scène, Anne vit dans la détresse pendant dix ans, « [p]eur de déranger, peur de [se] faire rabrouer, peur de regarder les choses en face. De [s]'entendre dire la vérité et d'avoir à accepter les conséquences. D'avoir à recommencer [sa] vie encore une fois, seule, avec un enfant de plus. Peur de la [rupture] » (313). Dans un état d'instabilité, elle s'accroche à la présence, si minime soit-elle, d'Amédée pour éviter de se retrouver «seul[e] à seul[e] directement face à [son] propre être au monde»(Landowski 1997 131). Pendant sa «[g]uérison intérieure excessivement lente et seulement partielle »(Andersen 339), Anne existe quelque part entre la vie et la mort, et le temps passe. Elle écrit sa peur, «[p]eur de la chute, de la fin, de la décrépitude » (22). L’incertitude au sujet de l'avenir est angoissante pour ce personnage qui se retrouve à un carrefour déconcertant et qui est à la recherche constante d'ordre et de stabilité. Selon ses souvenirs, ce besoin n'est jamais assouvi dans ses relations amoureuses et cela la frustre dans sa quête du sens de soi. Sa relation avec Amédée est celle qui la pousse à toucher le fond de ses passions et aussi de sa dégradation. Au moment de la narration, elle écrit : «Depuis dix ans, depuis qu'il a quitté la maison, je ne cesse de penser quotidiennement à lui » (295). L'absence de cet homme demeure une plaie qui ne s'est toujours pas cicatrisée. En se remémorant son désespoir le soir du départ d'Amédée, la nuit qu'elle a passée à l'attendre sur le seuil de l'appartement d'une autre, elle explique qu' «[e]lle pleurera ces mêmes larmes dix ans plus tard, à Bourg-la-Reine, elle sera de nouveau sur ce même palier, avec la même douleur » (318). Ces souvenirs déclenchent la profonde souffrance qui persiste malgré le passage du temps. L'état d'instabilité psychique dans lequel se retrouve Anne au moment de l'écriture provoque un besoin d'introspection dans le but de se libérer de ses souvenirs angoissants pour pouvoir ensuite poursuivre une croissance saine.

Dans Étrangers à nous-mêmes, Kristeva s'appuie sur les théories de Freud, pour expliquer que «l'inquiétante étrangeté se produit lorsque s'effacent les 'limites entre imagination et réalité" », puisque toute forme d' «incertitude intellectuelle » est caractérisée par «un affaiblissement de la valeur des signes en tant que tels et de leur logique propre » $(275,278)$. Cet état de déperdition fait en sorte que «le signe n'est pas vécu comme arbitraire, mais prend une importance réelle. En conséquence, la réalité 
matérielle que le signe devrait couramment indiquer s'effrite au profit de l'imagination » (27). Tout ce que l'être peut s'imaginer comme étant dangereux et déplaisant en lui, en l'absence de frontières entre la réalité et l'imaginaire, prend pour lui un sens particulier et produit un déséquilibre psychique qui doit être pris en compte pour assurer son bien-être. Cette altérité déroutante se présente également dans la littérature. Selon Kristeva, «[1e] choc de l'autre, l'identification du moi avec ce bon ou mauvais autre qui viole les limites fragiles du moi incertain, seraient donc à la source d'une inquiétante étrangeté dont l'aspect excessif, représenté en littérature, ne saurait cacher la permanence dans la dynamique psychique 'normale'» (278). C'est dans ce sens que dans l'acte de la remémoration narrative, le moi du passé puisse paraître étrange et déconcertant au moi incertain du présent. Dans l'écriture autofictive, caractérisée par le brouillement des frontières entre le symbolique et le référentiel, les effets de l'absence de l'autre sur le moi écrivant s'avèrent d'autant plus révélateurs.

Après la rupture avec Amédée, Anne s'arme de l'écriture pour affronter l'absence de son bien-aimé et contempler son propre être du passé. Par la remémoration, elle constate que son troisième mariage l'a empêchée d'être présente pour ses parents lorsqu'ils ont eu le plus besoin d'elle, et cela ajoute à son abjection. Au moment de l'écriture, elle envisage, véritablement seule, le vide créé par l'absence d'Amédée et celui de ses parents, ce qui la propulse vers l'étrangeté présente en elle-même et la reconnaissance de ses manquements auprès de sa famille, surtout de ses parents qui ont su la soutenir et la protéger à travers son enfance, ses mariages, et ses exils ${ }^{6}$, ainsi qu'auprès de ses enfants qui ont été victimes de ses choix. Son récit fragmentaire lui permet d'exposer et d'examiner son moi du passé, dans ses gestes les plus nobles et les plus vils.

En traduisant son désarroi en français, langue qui n'est celle d'aucun membre de sa famille (31), Anne peut s'exprimer plus ouvertement et réduire le risque de la critique sociale et surtout familiale ${ }^{7}$, tout en s'appropriant un lieu d'écriture dont elle tire un sens d'appartenance privilégié. Comme l'affirme Kristeva, il est possible, en utilisant un nouvel idiome, de «devenir virtuose avec ce nouvel artifice qui vous procure d'ailleurs un nouveau corps [...] Vous avez le sentiment que la nouvelle langue est votre résurrection $[\ldots] »(27)$. Il est plausible d'affirmer que la langue dans laquelle Anne 
choisit d'écrire est aussi un moyen pour elle de se rapprocher de ses lecteurs et lectrices, groupe de référence/narrataires potentiellement plus compatissant que celui de son passé. Ce groupe a une identité qu'elle imagine être homologue à la sienne, lectrice francophone avide d'aventure et d'évasion ${ }^{8}$. Selon Cécilia W. Francis, «il s'agit d'instaurer une relation désirante faisant intervenir des modalités telles la manipulation, la séduction et la capture » (2005-2006, 9). Cette recherche d'un groupe de référence auquel elle aspire s'associer et avec lequel elle veut établir « une relation désirante » est rattachée au projet d'écriture d'Anne qui lui permet de se rapprocher de deux groupes nouveaux : les auteurs français à qui elle se lie en écrivant ${ }^{9}$ et les narrataires francophones à qui elle s'adresse.

\section{Les relations intersomatiques}

Dans Passions sans nom, Landowski se penche sur « une théorie du sens » (2004, 19) qui implique non seulement la psyché de l'être, mais tout son corps. Il s'intéresse aux interactions « où la génération du sens passe par les modulations du rapport esthétique [qui] implique d'emblée et l'objet, envisagé du point de vue de ses qualités sensibles intrinsèques, et un sujet à même non seulement de les percevoir, et éventuellement de les décrire, mais aussi (ou d'abord) d'en éprouver les effets signifiants, en acte » (305). Cela implique des interactions qui dépassent l'ordre intersubjectif, qui tiennent compte de l'esthétique et qui prennent une forme intersomatique. Chaque corps vivant et interagissant participe à l'actualisation « de nouvelles configurations signifiantes » (304). Ainsi, le corps a son rôle à jouer en tant que lieux de mémoire et c'est un aspect important de l'œuvre d'Andersen. Selon Anne Muxel,

Le corps dit tout, se souvient de tout, et cette mémoire là serait souveraine, car imprimée au plus profond, au tréfonds de soi-même. [... Si] le corps oublie peu, si tout s'y inscrit, s'il est mémoire, cette mémoire reste sans doute la plus inaccessible, la plus difficile à toucher, à retrouver, et surtout à conscientiser. Parler le corps c'est presque tout dire, y compris ce que l'on ne sait pas ou mal. [...] Parler le corps, c'est se livrer tout entier. (116-117)

Nous voyons chez la narratrice, un désir de faire parler son corps et, ce faisant, de libérer tout souvenir qu'il soit source de nostalgie ou d'angoisse. Le sens accordé à ces souvenirs inscrits au lieu le plus profond, le plus intime de l'être est important puisque ceux-ci sont fondés sur les expériences intersomatiques du sujet. En «parlant le corps », on évoque 
nécessairement l'autre et son effet sur soi. Pour la première fois, la narratrice raconte que son beau-frère Hermann l'a agressée sexuellement alors qu'elle était adolescente et qu'elle gardait son enfant. Peu après, Mathias, le frère aîné de Hermann, profite d'une occasion semblable pour la violer. Pour ne pas perturber «l'apparent calme familial» (55), ces deux moments marquants dans la vie de la narratrice sont tus pendant quarante ans, jusqu'au moment de l'écriture de son texte. Elle explique :

À force de vouloir être adulte, à force de vouloir croire qu'il s'agissait d'actes accomplis par moi, j'ai permis à ces deux faux frères de s'en tirer à bon compte. [...] Je le raconte aujourd'hui dans l'intérêt de la totalité de mon existence, sachant très bien que certains m'accuseront de rêveries, de fantasmes, de dévergondage, d'imagination maladive. (55)

Ainsi, elle ne peut plus s'empêcher d'accuser ses agresseurs et révèle les deux viols dans le but de se réapproprier son corps ravalé.

Selon les souvenirs d'Anne, son corps est gêné par les hommes à qui elle fait confiance, mais elle découvre que l'écriture est une forme d'expression qui lui permet d'explorer ses passions en toute sécurité, de façon à contribuer à son orientation vers le bien et à son adaptation. Dans l'écriture de son être en devenir, elle s'expose corps et âme dans le but de se réapproprier son corps avili, bien que ce geste éveille sa crainte de «souiller [sa] famille, [ses] amis, le monde en général»(30), et cela signale, en termes landowskiens, sa prise de conscience de la position qu'elle adopte en relation aux divers groupes de référence, soit à l'autre, ainsi que la position dans laquelle elle risque d'être placée par ceux-ci à cause de l'acte d'écriture (voir Landowski 1997, 59).

Dans «Avant A. ou vingt-huit questions » (228), Anne avoue qu' «[elle trouverait] utile de parler [d'elle-même], de [son] corps amoureux, aux paroles des autres [elle voudrait] ajouter la [sienne]»(237). Un questionnaire sur la sexualité féminine qu'elle trouve dans F Magazine sert d'encadrement à cette réflexion sur sa vie amoureuse avant la rencontre d'Amédée, ce qui explique le titre de cette partie du l'œuvre. Ici, la narratrice entre en dialogue avec un texte, illustrant la dynamique intersomatique déclenchée par la lecture. En parlant de sa sexualité, Anne parle de son corps ainsi que de l'acte d'écriture et de l'effet que les hommes ont eu sur sa dignité. À la suite de son « dépucelage à quinze ans »(250), elle décrit quelques aventures d'adolescence qui l'amènent à renoncer à son corps et à ses rêves, un repli sur elle-même qu'elle cherche toujours à comprendre (252). Tout en se croyant vouée à une existence rangée, Anne 
préfère le faire « en dehors du cercle de la famille » (252). C'est alors qu'elle rencontre son premier époux, Paul, à Ahrenshoop. «Prisonnière » (254), « objet du désir impuissant d'un homme» (257), Anne ne trouve aucun bonheur dans ce mariage stérile et, malgré les menaces de Paul et du père de celui-ci, elle quitte son mari, retrouvant ainsi le désir de vivre sa vie (260). Dans l'écriture, l'intention de la narratrice suit celle de ses gestes passés : «Il faut que mon corps secoue cet homme allongé sur moi, il faut que je me défasse de lui, il faut que je sorte de mon corps et de moi-même les mots pour dire ce qui nous est arrivé, pour que nous soyons purifiés, lavés, comme au hammam, libérés » (253-254). Le corps discursif lui permet donc de se dégager du corps oppressif masculin. Grâce au récit, les corps du couple sont libérés des souvenirs pétrifiants de la narratrice.

Après le divorce, Anne pense commencer «la vraie vie » (260) mais, en 1943, c'est la guerre et elle doit se soumettre à « la servitude » (260) du service militaire avant de pouvoir poursuivre ses études. Pendant les deux années qui suivent, son ${ } \operatorname{CORPS}^{10}$ est mis AU TRAVAIL» (260) et «MENACÉ de destruction» (262). Elle se retrouve encore une fois dans un état de renonciation, où «l'indépendance n'est plus essentielle. La guerre et ses morts [1]'ont vaincue [elle] aussi, [son] corps fort de sa jeunesse et de sa santé est un CORPS VAINCU qui s'accrochera au premier vainqueur venu » (266). Corps et rêves de nouveau abandonnés, Anne se marie pour la deuxième fois. Si Paul promettait une réconciliation avec le lieu maternel d'Ahrenshoop, Pierre, un soldat français d'origine tunisienne, lui offre l'occasion de se rapprocher du lieu natal de son père, l'Afrique mythique qu'elle s'imagine depuis l'enfance. (66) Dans ce deuxième mariage, Anne découvre deux nouveaux états corporels : son «CORPS AFFAMÉ » (268) et son «CORPS MATERNEL»(270). D'abord, la pauvreté lui apprend une nouvelle façon d'être, l'oblige à se préoccuper constamment de la nourriture et d'entreprendre des tâches accablantes. Mais ce n'est pas seulement la nourriture qui fait défaut. Ce mariage manque d'intimité. L'acte sexuel initié quotidiennement par Pierre pour affirmer sa virilité n'est supporté par son épouse que dans le but d'assurer la paix conjugale. Anne explique : «Aucun orgasme dans ces conditions-là, aucun désir, j'étais comme prostituée, je payais cher mon erreur d'épouser Pierre au lieu de trouver le courage d'élever seule mon enfant, quitte à le priver de son père, à violer finalement l'image sacrée de Vater, Mutter, Kind» (270). Après la naissance d'un deuxième enfant conçu 
dans la contrainte, Anne constate que «ce n'est pas en les mettant au monde [qu'elle a] cessé de souffrir pour eux, dans [son] corps » (271). Devant son époux violent, Anne se sent impuissante : «Mes enfants devant moi battus, mes enfants à moi volés, mes enfants et moi prisonniers, et moi si longtemps incapable de les défendre » (275). Son corps affaibli par cette lutte constante, elle quitte la Tunisie à vingt-huit ans et rentre chez ses parents à Berlin, laissant ses enfants aux soins d'un «père dément » (271), le temps qu'elle reprenne ses forces. Son état physique fragilisé l'empêche d'atteindre un sens de plénitude en tant que femme et mère et devant cet échec, elle divorce une deuxième fois. Heureusement, elle est réconciliée avec ses enfants avant de partir pour le Canada, cherchant encore une fois à s'éloigner d'un milieu qu'elle supporte mal. Elle constate par contre que son «CORPS DÉPLACÉ » (285) n’est pas à l'abri des souvenirs.

Les relations amoureuses sont des relations intersomatiques privilégiées dans la construction du sens. Les souvenirs d'Anne montrent que les êtres choisis comme compagnons de vie ont eu un effet négatif sur sa dignité et ont affecté son être au monde. Anne se souvient de certaines critiques et en anticipe d'autres : «Trop sérieuse, trop intense, trop maternelle. Trop étroite, disait Paul [son premier époux]. Trop habituée au luxe et à la dépense, disait Pierre [son deuxième époux]. Trop vieille, trop laide, trop grosse, trop énergique, trop décisive, dirait Amédée [son troisième époux] » (278). Anne se questionne sur les messages reçus des hommes qui l'entourent : «N'ai-je pas été, toute ma vie durant, hystérique, nymphomane, à la recherche du divertissement ?» (292). Elle méprise le fait qu'elle se soit minée en cherchant à leur plaire : «Je me suis éparpillée, gaspillée, j'ai été larve rampante d'un acte à l'autre, gluante je me suis attachée à telle branche ou à telle autre, mon arbre était le pénis, je le dévorais tout en faisant des mouvements désespérés pour me défaire de ce qui m’empêchait de devenir sinon une femme aimable, du moins un insecte agréable à regarder »(292). Il s'agit ici d'une dialectique maître-esclave fondée sur la domination de l'un et la soumission de l'autre. Comme l'explique Kristeva, en s'appuyant librement sur la philosophie de Hegel, la soumission est, en fait, un masque, une « peau anesthésiée dont [1'esclave] s'enrobe pour se procurer une cachette où il jouit de mépriser les faiblesses hystériques de son tyran » (16). L'écriture procurera enfin à Anne un endroit où se réfugier pour détailler les abus dont elle a été victime. Elle se retrouve dans un état de déséquilibre psychique pendant 
lequel elle se sent seule devant elle-même. Dans cet état d'esprit, elle se réfère à ce que dit l'autobiographie de son père, au sujet de l'acte d'introspection :

[Werner] explique, à la page 91, qu'il y a en chacun de nous un autre, qui observe froidement nos petites actions et les mesure à l'aide d'un mètre éternel. Je voudrais faire de mon autre une camarade, lui faire jeter son mètre, devenir une avec elle. Mais, distante, elle continue à me juger, elle ne me rejoint pas dans la multitude de mes souvenirs, à la place centrale que j'occupe dans cet amas confus de personnages étranges, y compris mes trois enfants. (292-293)

Cette camarade, qui pourrait accompagner Anne sur le chemin de sa vie, viendrait remplacer la figure normative traditionnelle devant laquelle elle cherchait à se mesurer, sans jamais se trouver à sa hauteur. Anne cherche à atteindre un état de paix et d'autonomie. Elle reconnaît que les conceptions morales qu'elle a acquises de sa famille, surtout liées à ce que représente une vie comblée, ne la satisfont pas. Elle se propose de « [d]evenir [son] propre père, [sa] mère, [son] enfant » (341). C'est à travers l'écriture qu'Anne assume son être, passé et présent, en toute sa complexité.

\section{Faire de son autre une camarade bienveillante}

À la fin du roman, après avoir complété l'exercice de la narration de son passé, Anne se scrute dans le miroir. C'est une activité qu'elle évite depuis longtemps, car cet objet s'associe à l'effet nuisible qu'avait eu sur elle le regard d'Amédée (313). Si elle avait eu peur de jeter un regard rigoureux sur sa vie, Anne peut maintenant, grâce à l'écriture, envisager son être du passé, le reconnaissant comme autre, et anticiper une période de sa vie dans laquelle «l'avenir 'rachète' le passé [qui fait] partie du récit d'une vie qui [a] un sens et une raison d'être » (Taylor 76). Devant son image réfléchie, elle remarque : «Miroir. Glace. Froide. [...] Introspection. Tu ne t'es pas occupée de ta mère malade, tu n'as pas su t'occuper comme il faut de ton premier enfant, ni du deuxième, même si c'était un peu mieux. Tu essaies pour la dernière, la troisième. Tu essaies, tu ne réussis pas toujours. Et tu veux réussir. [...] Es-tu bonne, Anne ? » (Andersen 343). Dans l'acte mémoriel, Anne constate qu'elle s'est rendue minable à ses propres yeux et souvent aux yeux des membres de sa famille. Sa narration lui permet de reconnaître les fautes commises dans sa quête du bonheur, attestant de la nature morale de son introspection alors que son orientation vers le bien se concrétise : 
Aime-toi, puisqu'il faut que tu vives avec toi, ton visage, ta vie sage, ta vie folle. [...] Le visage abject n'a jamais été dans ce miroir. Dans l'ensonge seulement, dans mes rêves j'avais ce visage d'horreur. L'horreur envisagée. Le rêve où je tue. [...] Je m'envisage. Je me souris. Je ne suis pas horrible. La réalité est vivable. Je n'ai pas une tête à faire peur, ni aux bébés ni aux hommes... Je peux vivre encore. [...] J'aime mes enfants, mes morts, mes vivants, moi. (343-344)

Ayant affronté l'inquiétante étrangeté dans son écriture, elle peut maintenant reprendre sa vie. Devant la glace, elle veut devenir pour elle-même cette camarade bienveillante, se distanciant de son moi du passé.

Le roman se termine sur «La fête » (345), qui présente un lieu féminin collectif utopique où il serait possible pour la narratrice d'évoluer en toute sécurité affective. Anne quitte le lieu sécurisant «dans le fini »(27) du cadre de ses souvenirs, marquant sa transformation de «femme rompue » à «femme sans forme imposée » (22). Cette fête a lieu au Canada et les invitées sont exclusivement des femmes. Toujours à la recherche d'un lieu d'appartenance, Anne se crée un lieu où il est possible de « [se] paterner, [se] materner, [s]'enfanter tous les jours » (341). La célébration féminine se fait autour d'un pommier qui remplace maintenant son «arbre [du passé, qui] était le pénis » (292). Les femmes dans ce lieu utopique se soutiennent, s'entraident et célèbrent le bonheur. Kristeva souligne l'importance du repas partagé qui sert de rencontre entre le passé remémoré, le présent vécu et les projets d'avenir :

La rencontre commence souvent par une fête de la bouche : du pain, du sel et du vin. Un repas, communion nutritive. [...] Mais ce coin de table plaisamment dévorant est parcouru des chemins de la mémoire : on se souvient, on projette, on récite, on chante. Le banquet, nourricier et initialement quelque peu animal, s'élève aux fumées des songes et des idées : les fêtards de l'hospitalité s'allient pour quelque temps aussi par l'esprit. Miracle de la chair et de la pensée, le banquet de l'hospitalité est l'utopie des étrangers [...]. (22-23)

Comme il s'agit justement dans le roman d'Andersen d'utopie, les femmes assemblées peuvent «célébrer sans contrainte une fois pour toutes la possibilité du bonheur» (Andersen 348). Pour Anne, « [a]yant achevé le récit », le «plan de fête » consiste à «ne plus se raconter. Se défaire de la forme sans pour autant la perdre. S'imaginer tout en restant réelle » (348). Cela marque une réconciliation de la narratrice et de son moi et suggère que l'œuvre artistique et littéraire peut servir à inspirer le bonheur et la paix dans 
la complicité féminine ${ }^{11}$. À la fin du roman, l'identité de l'individu s'intègre dans la joie à celle de la collectivité des femmes.

Chez Marguerite Andersen, vivre et écrire sont deux activités qui se complètent. Sa vie est ponctuée d'écriture, quelquefois à des moments où le besoin de se réorienter est urgent, tant pour ses protagonistes que pour elle. Comme c'est le cas pour l'auteure, Anne cherche à trouver le fil narratif de son identité et à créer un nouveau lieu d'appartenance qui mène à la catharsis. Il s'agit d'un exercice d'organisation narratif qui reflète les regrets d'Anne au sujet de ses relations passées, son sentiment de solitude et son désir de se redéfinir pour pouvoir poursuivre son cheminement. À la fin, le banquet utopique semble résoudre ce que Landowski qualifie d'inquiétude de la part d'un Nous en construction, en substituant au Nous individuel un Nous collectif (1997 42). Il y a une nouvelle façon de vivre : «Se toucher. S'aboucher. S'entrelacer. Se serrer. Se caresser de tous les doigts de fée. Ne faire qu'une»(Andersen 350). Anne, qui avait souffert de l'absence de ses parents, de son manque d'intimité avec ses maris successifs, et surtout de sa profonde solitude face à l'autre, a réussi, grâce à l'écriture, à se définir et à inventer une communauté de femmes auxquelles elle peut s'identifier.

\section{Bibliographie}

Andersen, Marguerite. De mémoire de femme. Ottawa : L'Interligne, 2002. (Première édition : Quinze, 1982).

---. «Subversive Texts : Québec Women Writers ». Studies in Canadian

Literature / Études en Littérature Canadienne 13.2 (1988) : 139.

Deneys-Tunney, Anne. Écritures du corps : De Descartes à Laclos. Paris : PUF, 1992.

Doubrovsky, Serge. Autobiographiques : de Corneille à Sartre. Paris : PUF, 1988.

Francis, Cécilia. «La subjectivité en mouvance : présentation ». Recherches sémiotiques / Semiotic Inquiry 25.3, 26.1 (2005 2006) : 9.

Gasparini, Philippe. Est-il je ? Paris : Seuil, 2004.

Hoover Braendlin, Bonnie. «Alther, Atwood, Ballantyne, and Gray : Secular Salvation in the Contemporary Feminist Bildungsroman ». Frontiers 4.1 (1979) : 18.

Hotte, Lucie. Préface. De mémoire de femme. De Marguerite Andersen. Ottawa: L'Interligne, 2002. 7-12. 
Huston, Nancy. L’Espèce fabulatrice. Arles : Actes Sud, 2008.

Kadar, Marlene, dir. «Coming to Terms : Life Writing - from Genre et Critical Practice ». Essays on Life Writing : From Genre to Critical Practice. Toronto : U of Toronto P, 1992, 3-16.

Kristeva, Julia. Étrangers à nous-mêmes. Paris : Fayard, 1988.

Landowski, Eric. Passions sans nom. Paris : PUF, 2004.

---. Présences de l'autre. Essais de socio-sémiotique II. Paris : PUF, 1997.

Lejeune, Philippe. «Le journal comme ‘antifiction’ ». Poétique 149 (2007) : 3-14.

Muxel, Anne. Individu et mémoire familiale. Paris : Nathan, 1996.

Olney, James. Memory \& Narrative. Chicago : U of Chicago P, 1998.

Ong, Walter. « The Writer's Audience Is Always a Fiction ». Contemporary Literary

Criticism. Dirs. Robert Con Davis et Ronald Schleifer. $2^{\mathrm{e}}$ éd. New York :

Longman, 1984. 82-99.

Saul, Joanne. Writing the Roaming Subject : The Biotext in Canadian Literatures.

Toronto : U of Toronto P, 2006.

Taylor, Charles. Les Sources du moi : La formation de l'identité moderne. Trad.

Charlotte Melançon. Montréal : Boréal, 2003.

\footnotetext{
Notes

${ }^{1}$ Sans doute y a-t-il aussi des éléments autobiographiques dans d'autres livres de Marguerite Andersen. Seule une connaissance approfondie de la vie de l'auteure permettrait de tous les relever.

${ }_{2}^{2}$ Pour arriver à cette conclusion, nous avons consulté les ouvrages de Serge Doubrovsky, Philippe Gasparini, Marlene Kadar, Philippe Lejeune, James Olney et Joanne Saul qui se trouvent dans la bibliographie.

${ }^{3}$ Landowski l'explique ainsi : le sens est une fonction «indéfiniment en construction» (2004 19), qui «ne peut naître que dans une rencontre où le sujet se trouve d'abord tout entier mis à l'épreuve, presque au défi, de vivre la présence sensible de l'autre, du monde, de l'objet (et à la limite même, celle de son propre corps) comme faisant sens ; il faut que le sujet trouve par rapport à la configuration sensible que le monde lui offre une manière de s'ajuster, telle que puisse en émerger, pour lui, du sens et de la valeur » (8).

${ }^{4}$ Le Landtag est l'assemblée représentative d'un état allemand.

${ }^{5}$ Selon le récit d'Anne, elle héberge Amédée et rédige, pour lui, son mémoire de maitrise et sa thèse de doctorat. De plus, Anne explique plus tard qu'il l'a trompée tout au long de leur relation et elle constate qu'il a miné son estime de soi. Le récit de Dominique en est le témoignage.

${ }^{6}$ Notons aussi qu'Anne regrette une certaine lâcheté qu'elle s'attribue quant à l'Holocauste: «J'étais coupable de ne pas avoir résisté, d'avoir ri, mangé, fait l'amour pendant que d'autres souffraient. [...] j'ai pu quitter l'Allemagne en novembre 1945, quitter mon pays que je n'aimais plus. Et cela non plus ne constituait aucunement une action héroïque : j'ai par ce départ évité toutes les difficultés de l'aprèsguerre $\gg(89-90)$.

${ }^{7}$ Ceci se confirme vingt-deux ans plus tard dans Parallèles.
} 
${ }^{8}$ Selon Walter Ong, dans son étude «The Writer's Audience Is Always a Fiction », «the writer must construct in his imagination, clearly or vaguely, an audience cast in some sort of role - [...] reflective sharers of experience, [among others] » (87). De plus, il précise que le lecteur ou la lectrice «must correspondingly fictionalize itself. A reader has to play the role in which the author has cast him, which seldom coincides with his role in the rest of actual life » (87).

${ }^{9}$ Ce projet est clairement annoncé par des textes en exergue signés Sartre, Duras, Baudelaire et Tournier et par les références à Stendhal, Flaubert et Balzac dans le texte.

${ }^{10}$ Les mots écrits en lettres majuscules correspondent aux mots qui sont placés dans le casier d'imprimerie qu'Anne utilise pour classer ses souvenirs.

${ }^{11}$ Cette fête rappelle deux autres œuvres : «The Dinner Party » (1974-1979) de Judy Chicago, exposé au Musée d'art contemporain de Montréal, de mars à mai 1982, et au Musée des beaux-arts de l'Ontario, de mai à juillet 1982 ; et Pique-nique sur l'Acropole de Louky Bersianik (1979). Dans un article, Marguerite Andersen explique : «Joy is one of Bersianik's inspirations. In Pique-nique sur l'Acropole, she brings women who do not have the money for a banquet to an outdoor picnic. Here they will joyfully discuss their sexuality in all its multiplicity, speaking in a choir of multiple voices of the female body as a source of creativity, with the skin and its pores/holes being receiver and giver of female speech, touch, and pleasure » (Andersen, 1988, 139). 